\title{
Path independent integrals to identify localized plastic events in two dimensions
}

\author{
Mehdi Talamali ${ }^{1}$, Viljo Petäjä ${ }^{1}$, Damien Vandembroucq ${ }^{1}, 2$ and Stéphane Roux ${ }^{3}$ \\ 1: Unité Mixte CNRS/Saint-Gobain "Surface du Verre et Interfaces" \\ 39 Quai Lucien Lefranc, \\ 93303 Aubervilliers cedex, FRANCE \\ 2: Laboratoire PMMH, ESPCI/CNRS/Paris 6/Paris 7 \\ 10 rue Vauquelin, 75231 Paris cedex 05, France \\ 3: LMT-Cachan \\ ENS de Cachan / CNRS-UMR 8535 / Université Paris 6/PRES UniverSud \\ 61 avenue du Président Wilson, \\ F-94235 Cachan Cedex, France.
}

\begin{abstract}
We use a power expansion representation of plane elasticity complex potentials due to Kolossov and Muskhelishvili, to compute the elastic fields induced by a localized plastic deformation event. Far from its center, the dominant contributions correspond to first order singularities of quadrupolar and dipolar symmetry which can be associated respectively to pure deviatoric and pure volumetric plastic strain of an equivalent circular inclusion. Constructing holomorphic functions from the displacement field and its derivatives, it is possible to define path independent Cauchy integrals which capture the amplitudes of these singularities. Analytical expressions and numerical tests on simple finite element data are presented. The development of such numerical tools is of direct interest for the identification of local structural reorganizations which are believed to be the key mechanisms for plasticity of amorphous materials.
\end{abstract}

\section{INTRODUCTION}

Plasticity of amorphous materials has motivated an increasing amount of studies in recent years. In the absence of underlying crystalline lattice in materials such as foams, suspensions or structural glasses, it is generally accepted that plastic deformation results from a succession of localized structural reorganizations 1, 2, 3, 4. Such changes of local structure release part of the elastic strain to reach a more favorable conformation and induce long range elastic fields. The details of such local rearrangements and of the internal stress they induce obviously depend on the precise structure of the material under study, and its local configuration . However, the important observation is that outside the zone of reorganization, a linear elastic behavior prevails. Therefore, elastic stresses can be decomposed onto a multipolar basis and independently of the material details, it is possible to extract singular, scale-free, dominant terms which can be associated to a global pure deviatoric or pure volumetric local transformations of an equivalent circular inclusion. In particular, the elastic shear stress induced by a localized plastic shear exhibits a quadrupolar symmetry. This observation has motivated the development of statistical models of amorphous plasticity at mesoscopic scale based upon the interaction of disorder and long range elastic interactions 8, 6, 7, 8. In the same spirit statistical models were also recently developed to describe the plasticity of poly-crystalline materials [9]. Several numerical studies have been performed recently to identify these elementary localized plastic events in athermal or molecular dynamics simulations of model amorphous material under shear [10, 11].

The question remains how to identify and analyze these transformation zones. In analogy with the path independent Rice J-integral 12] developed to estimate the stress intensity factor associated to a crack tip stress singularity, we aim here at capturing the stress singularity induced by the local plastic transformation which can be treated as an Eshelby inclusion[13. In two dimensions, we develop a simple approach based upon the Kolossov-Muskhelishvili (K\&M) formalism of plane elasticity 14]. This is an appealing pathway to the solution since these zones will appear as poles for the potentials, and hence Cauchy integrals may easily lead to contour integral formulation which are independent of the precise contour geometry, but rather rely on its topology with respect to the different poles which are present.

Although these techniques have been mostly used in the context of numerical simulations in order to estimate stress intensity factors from finite element simulations, they are now called for to estimate stress intensity factors from experimentally measured displacement field from e.g. digital image correlation techniques. In this case, interaction integral techniques 15] or least square regression 16] techniques have been applied. Noise robust variants have also been proposed[17. These routes could also be followed in the present case.

Though the present work is restricted to two dimensions due to the complex potential formulation, similar questions can be addressed for the three dimensional version of this problem using the same strategy but a different methodology. In the following, we briefly recall the K\&M formalism, and we give analytic expressions of contour integrals allowing to capture the singular elastic fields and we present a few numerical results based on a finite element simulation supporting our analytical developments. 
In Section 2, we present the theoretical basis of our approach in terms of singular elastic fields, while Section 3 introduce the contour integral formulation. In section 4, a numerical implementation based on finite element simulations is presented together with the results of the present approach. This application allows to evaluate the performance and limitations of the contour integral procedure and check the detrimental effect of discreteness. Section 5 presents the main conclusions of our study.

\section{POTENTIAL FORMULATION}

In two dimensions the Kolossov and Muskhelishvili potentials (K\&M) can be used to write the elastic stress and displacement fields $\mathbf{U}$ and $\sigma\left[14\right.$. Using a complex formulation, we introduce the elastic displacement $\mathbf{U}=U_{x}+i U_{y}$ and the stress tensor field through two functions, the real trace $S_{0}=\sigma_{x x}+\sigma_{y y}$ and the complex function $\mathbf{S}=$ $\sigma_{y y}-\sigma_{x x}+2 i \sigma_{x y}$. In the framework of linear elasticity, balance and compatibility equations can be rewritten as:

$$
\begin{aligned}
S_{0, z}-\mathbf{S}_{, \bar{z}} & =0, \\
S_{0, z \bar{z}} & =0,
\end{aligned}
$$

where $z=x+i y$ is the complex coordinate and the notation $A_{x}$ is used to represent the partial derivative of field $A$ with respect to coordinate $x$. Note that we assumed zero surface density force and that equation (2) is here the classical Beltrami equation which expresses the kinematic compatibility condition in terms of stress. The general solution to these equations can be obtained through the introduction of two holomorphic functions $\varphi$ and $\psi$, called the K\&M potentials. The displacement and the stress field can be written [14.

$$
\begin{aligned}
2 \mu \mathbf{U} & =\kappa \varphi(z)-z \overline{\varphi^{\prime}(z)}-\overline{\psi(z)}, \\
S_{0} & =2\left[\varphi^{\prime}(z)+\overline{\varphi^{\prime}(z)}\right], \\
\mathbf{S} & =2\left[\bar{z} \varphi^{\prime \prime}(z)+\psi^{\prime}(z)\right],
\end{aligned}
$$

where $\mu$ is the elastic shear modulus and $\kappa=(3-4 \nu)$ for plane strain and $\kappa=(3-\nu) /(1+\nu)$ for plane stress, $\nu$ being the Poisson's ratio.

\section{PLASTIC INCLUSION AND SINGULARITY APPROACH IN 2D}

\section{A. Singular terms associated to plastic inclusion}

This K\&M formalism can be applied to two-dimensional inclusion problems 18, 19. Let us consider the case of a small inclusion of area $\mathcal{A}$ experiencing plastic deformation and located at the origin of the coordinate system $z=0$. It is assumed that the stress is a constant at infinity. Outside the inclusion, the K\&M potentials can be expanded as a Laurent series as

$$
\varphi(z)=\alpha^{\text {out }} z+\sum_{n=1}^{\infty} \frac{\varphi_{n}}{z^{n}}, \quad \psi(z)=\beta^{\text {out }} z+\sum_{n=1}^{\infty} \frac{\psi_{n}}{z^{n}}
$$

The linear terms can be easily identified as corresponding to uniform stresses while constant terms (omitted here) would lead to a rigid translation. It can be shown in addition that the dominant singular terms $\varphi_{1} / z$ and $\psi_{1} / z$ can be associated to the elastic stress induced by the plastic deviatoric and volumetric of an equivalent circular inclusion of area $\mathcal{A}$. Namely, considering a circular inclusion experiencing a plastic shear strain $\gamma_{p}$ and a plastic volumetric strain $\delta_{p}$ we have 18 :

$$
\varphi_{1}=\frac{2 i \mu \mathcal{A} \gamma_{p}}{\pi(\kappa+1)}, \quad \psi_{1}=-\frac{2 \mu \mathcal{A} \delta_{p}}{\pi(\kappa+1)} .
$$

In particular, for a pure shear plastic event we obtain a quadrupolar symmetry:

$$
\sigma_{x y}=-\frac{2 \gamma_{p} \mu}{\kappa+1} \frac{\mathcal{A}}{\pi r^{2}} \cos (4 \theta)
$$

Note that we have in general to consider a complex value of $\gamma_{p}$ to include the angular dependence of the principal axis. In contrast, the amplitude $\psi_{1}$ is a real number (note that the imaginary part would correspond to a point-like torque applied at the origin). 


\section{B. Generic character of the expansion}

Because of the well-known property of Eshelby circular inclusion, the above expansion limited to the $\psi_{1}$ and $\phi_{1}$ terms only is the exact (outer) solution of a uniform plastic strain distributed in the inclusion, and vanishing stress at infinity. However, one should note that this result is much more general. Indeed, it is seen that the physical size of the inclusion does not enter into the solution but through the products $\mathcal{A} \gamma_{p}$ and $\mathcal{A} \delta_{p}$. Therefore, a smaller inclusion having a larger plastic strain may give rise to the very same field, provided the products remain constant. Therefore, one can consider the prolongation of the solution to a point-like inclusion (with a diverging plastic strain), as being equivalent to the inclusion.

Then from the superposition property of linear elasticity, a heterogeneous distribution of plastic strain $\gamma_{p}(x)$ in a compact domain, $\mathcal{D}$, will give rise to such a singularity with an amplitude equal to

$$
\left[\mathcal{A} \gamma_{p}\right]_{e q}=\iint_{\mathcal{D}} \gamma_{p}(x) d x
$$

and the same property would hold separately for the volumetric part. As a particular case, one finds a uniform plastic strain for an inclusion of arbitrary shape.

This is the key property that allows to capture the equivalent plastic strain of an arbitrary complex configuration, for the above mentioned application to amorphous media. In fact, this is even the only proper way of defining the plastic strain for a discrete medium such as encountered in molecular dynamics simulations. The far-field behavior of the displacement and stress field can be accurately modelled, and without ambiguity by a continuum approach, and thus the above result will hold. In contrast, locally, the large scale displacement of several atoms may render difficult the direct computation of the equivalent plastic strain experienced in such an elementary plastic event.

Let us however stress one difficulty: As the above argument ignores the details of the action taking place within the "inclusion", plasticity has to be postulated. However, a damaged inclusion, where the elastic moduli have been softened by some mechanism, or even a non-linear elastic inclusion at one level of loading would behave in a similar way as the above plastic inclusion. Obviously, to detect the most relevant physical description, one should have additional informations, say about unloading. If the above amplitudes remain constant during unloading, plasticity would appear appropriate. If the amplitude decreases linearly with the loading, then damage is more suited. Finally, if the amplitudes varies reversibly with the loading, non-linear elasticity might be the best description. Thus, although one should be cautious in the interpretation, local damage detection from the far field may also be be tackled with the same tools.

\section{Path independent contour integrals}

In two-dimensions, this multipole expansion formalism in the complex plane suggests to resort to contour integrals to extract the singularities. However the displacement field is not a holomorphic function and cannot be used directly for that purpose. The strategy of identification of the singularities $\varphi_{n}$ and $\psi_{n}$ thus consists of expressing the potentials from the displacement field and its derivatives in order to extract the singularities via Cauchy integrals. We now simply express the displacement field and its derivatives:

$$
\begin{aligned}
2 \mu U & =\kappa \varphi(z)-z \overline{\varphi^{\prime}(z)}-\overline{\psi(z)} \\
2 \mu U_{, z} & =\kappa \varphi^{\prime}(z)-\overline{\varphi^{\prime}(z)} \\
2 \mu U_{, \bar{z}} & =-\overline{\varphi^{\prime \prime}(z)}-\overline{\psi^{\prime}(z)} \\
2 \mu U_{, z \bar{z}} & =-\overline{\varphi^{\prime \prime}(z)} .
\end{aligned}
$$

This gives immediately:

$$
\begin{aligned}
\varphi^{\prime}(z) & =\frac{2 \mu}{\kappa-1}\left[\kappa U_{, z}+\overline{U_{, z}}\right], \\
\varphi^{\prime \prime}(z) & =-2 \mu \overline{U_{, z \bar{z}}}=-\frac{\mu}{2} \overline{\nabla^{2} U}, \\
\psi^{\prime}(z) & =-2 \mu\left[\overline{U_{, \bar{z}}}-\frac{\bar{z}}{4} \overline{\nabla^{2} U}\right] .
\end{aligned}
$$

Note that, except a multiplicative constant, the two last expressions are independent of materials properties. In light of the expansion (5) of $\varphi$ and $\psi$ in Laurent series, if an anti-clockwise contour integration is considered along a path 
$\mathcal{C}$, Cauchy residues can be formed as

$$
\begin{aligned}
\varphi_{n} & =\frac{i \mu}{4 \pi n(n+1)} \int_{\mathcal{C}} z^{n+1} \overline{\nabla^{2} U} d z \\
\psi_{n} & =\frac{i \mu}{\pi n} \int_{\mathcal{C}} z^{n}\left[\overline{U_{, \bar{z}}}-\frac{1}{4} \bar{z} \overline{\nabla^{2} U}\right] d z
\end{aligned}
$$

These expressions can thus be obtained from the sole knowledge of the displacement field, a quantity which can be accessed from experiments, or from atomistic simulations. In the case of first order singularities (see Eq.(7)), the residue term thus only depends on the local plastic deformation (size and amplitude of deformation) and on the Poisson's ratio $\nu$ of the material.

Reverting to Cartesian coordinates, where the contour is expressed as a function of the curvilinear abscissa $s$ as $(x(s), y(s))$, the above expression can be written

$$
\begin{aligned}
\varphi_{1}= & \frac{\mu}{8 \pi} \int_{\mathcal{C}}\left[-2(x y)+i\left(x^{2}-y^{2}\right)\right]\left[\left(U_{x, x x}+U_{x, y y}\right)-i\left(U_{y, x x}+U_{y, y y}\right)\right]\left[\frac{d x}{d s}+i \frac{d y}{d s}\right] d s \\
\psi_{1}= & \frac{\mu}{4 \pi} \int_{\mathcal{C}}\left[2[i x-y]\left[\left(U_{x, x}-U_{y, y}\right)-i\left(U_{y, x}+U_{x, y}\right)\right]\right. \\
& \left.-\left[i\left(x^{2}+y^{2}\right)\right]\left[U_{x, x x}+U_{x, y y}-i\left(U_{y, x x}+U_{y, y y}\right)\right]\right]\left[\frac{d x}{d s}+i \frac{d y}{d s}\right] d s .
\end{aligned}
$$

\section{NUMERICAL IMPLEMENTATION}

The ultimate goal of a such a method would be to analyze numerical results obtained from molecular dynamics simulations of amorphous plasticity where such local structural reorganizations are expected to take place. This obviously raises the question of a well defined method for writing the continuous displacement field from the data of the discrete displacements of particles 20 and more generally the question of the sensitivity to noise of the above expressions. The first point is beyond the scope of the present work and we leave it for later studies. We thus focus on the more restricted question of the numerical implementation and its efficiency in the case of artificially noise-corrupted displacement data.

The method relies on contour integrations of derived fields of the displacement. The latter point induces a priori a strong sensitivity to noise. To limit such effects, first and second derivatives are extracted via an interpolation of the local displacement field by polynomial functions of the spatial coordinates. Moreover, the path independence of the contour integrals allows to perform spatial averages. We explore in the following the efficiency of this method on noisy data.

\section{A. Numerical generation of elastic fields induced by plastic inclusions}

Displacement fields are computed numerically using a finite element code, with square elements and bi-linear shape functions $\{1, x, y, x y\}$. Plane stress conditions of two-dimensional elasticity are used. The domain is a $150 \times 150$ square. Stress free conditions are enforced all along the domain boundary. The Poisson's ratio of the material is $\nu=0.20$. Since no quantitative values of the stress are used, the value of the Young's modulus is immaterial.

A plastic strain is implemented at the scale of one single isolated element. Within this element, the strain is the sum of a plastic uniform strain chosen at will, and an elastic strain. The latter is computed by solving for the twodimensional elastic problem, insuring force balance and kinematic continuity at all nodes including the nodes of the plastic element. The chosen kinematics is too crude to solve at the scale of one single element the elastic problem with a good accuracy. However, remote from the inclusion, the elastic perturbation is well accounted for, and since all our computations are based on paths lying at a distance from the inclusion, the formalism should be applicable. A single element allows us to have a maximum ratio between inclusion and domain size. The price to pay for this crude local description is that the quantitative estimate of $\mathcal{A} \gamma_{p}$ and $\mathcal{A} \delta_{p}$ will differ slightly from the theoretical expectation. Nevertheless the path independence, (size, shape, center, ...) is expected to hold.

We limited ourselves to such a description in order to mimic the difficulties one may face when having to deal with discrete element simulations. Indeed, the chosen finite element shape functions do not allow us to use this description strictly speaking in order to compute second order differential operators on the displacement, since the gradients of the latter are not continuous across element boundaries. Therefore, a regularization will be called for, as detailed below.

The choice of a regular square lattice is obviously oversimplified compared with the case of the random lattices associated with atomistic simulations. However, as $x$ and $y$ directions are obviously equivalent for square elements and as linearity is preserved by the finite element formulation, this formulation should not introduce any breaking 


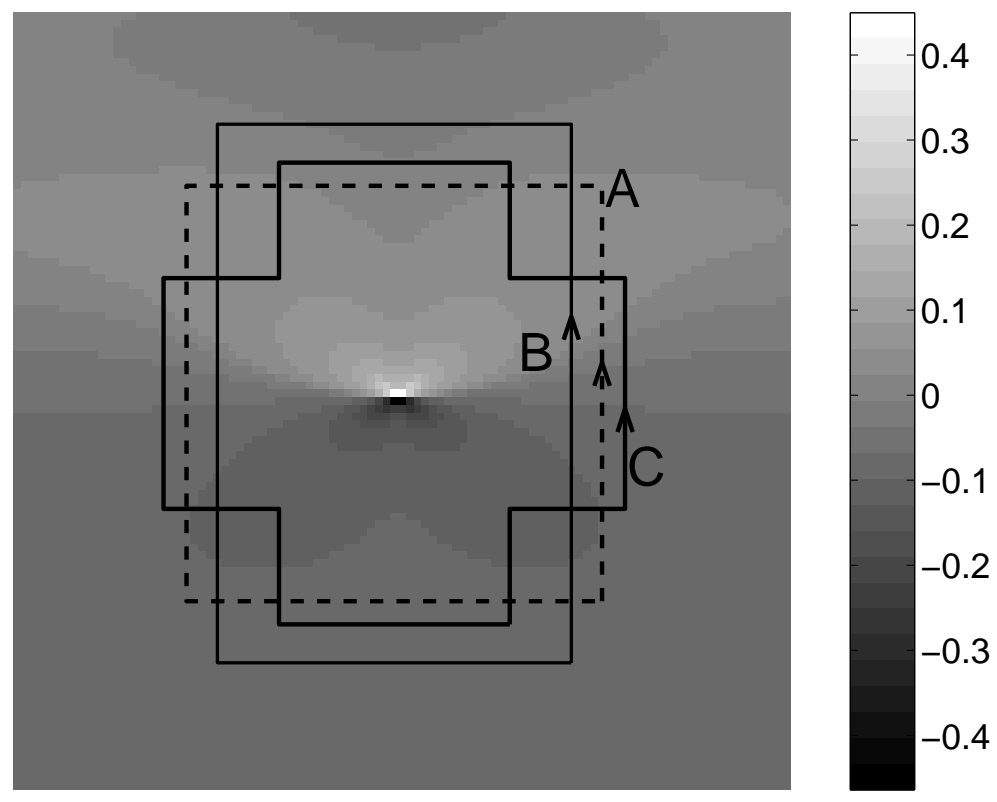

FIG. 1: Map of the displacement field $U_{y}$ induced by a plastic shear strain of a central square element. The oriented paths indicate (
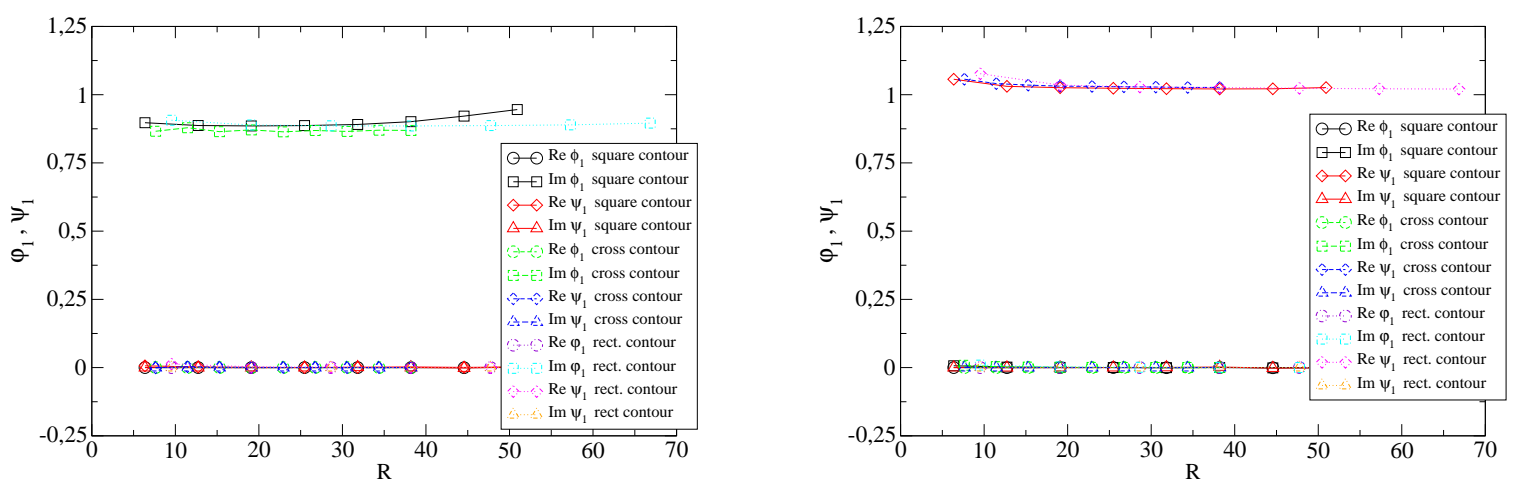

FIG. 2: Normalized values of numerical estimates $\varphi_{1}$ and $\psi_{1}$ obtained for three families of contours, respectively square, cross and rectangle shaped and of varying length $L$ in the case of a displacement field induced by the plastic shear strain (left) or contraction (right) experienced by the central element of a square lattice. Theoretical expectations are $\varphi_{1}=i, \psi_{1}=0$ (left) and $\varphi_{1}=0, \psi_{1}=1$ (right).

of symmetry. More specifically, the displacement field induced by a quadrupole of principal direction off axis can be obtained by a linear superposition of $x$ and $y$ components of the displacement field induced by a quadrupole aligned with the axis weighted by the sine and cosine of the quadrupole angle.

Finally, the finite size of the system is also a specific difficulty encountered in practice, whereas the above argument uses the assumption of an infinite domain. However, such a boundary condition should not induce additional poles within the domain, and can be considered as a common practical difficulty encountered for all practical use of this tool. All these arguments are possible causes of deviation from the theoretical expectation of path independence, and it thus motivates a detailed study of the method stability, robustness and accuracy.

Two test cases are studied. I: a central inclusion experiencing a shear strain $\gamma_{p}=1$ (because of linearity, the actual amplitude is meaningless) along the $x$-axis; II: a central inclusion experiencing a volumetric contraction $\delta_{p}=-1$. A map of the displacement fields $U_{y}$ in case $\mathrm{I}$ is given on Fig. 11. 


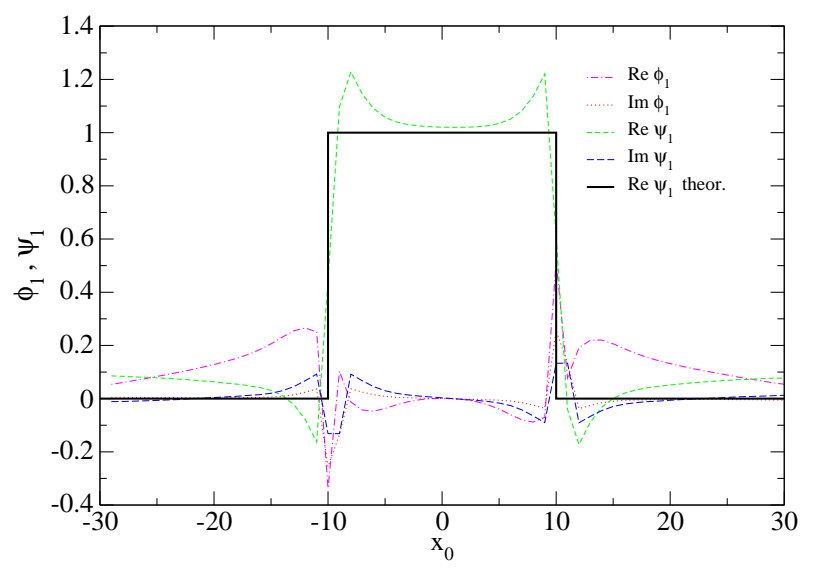

FIG. 3: Normalized values of numerical estimates $\varphi_{1}$ obtained for square contours of center $\left(x_{0}, 0\right)$ and of size $M=20$. The expected behavior of $\Re \psi_{1}$ (unity when the inclusion lies within the contour, zero otherwise) is represented by the bold line. Other quantities are expected to be zero.

\section{B. Interpolating displacement data}

The key ingredient is to go from a continuous but non-differentiable displacement field obtained from the finite element simulation to an evaluation of the second derivative at any point in the domain.

The results which are presented below have been obtained using the following procedure. A quadratic fit is performed on a square centered on one node to extract the first and second order derivatives, the obtained values are used to compute the integrals by quadrature. An alternative method has been tested: for an integration from $(x, y)$ to $(x+1, y)$, a simple fit is performed of the 12 nodes ranging from $(x-1)$ to $(x+2)$, and from $(y-1)$ to $(y+1)$, by the tensor product of polynomials $\left(1, x, x^{2}, x^{3}\right)$ and $\left(1, y, y^{2}\right)$ (12 functions). Then the integral of all required quantities can be computed. Estimates of derivatives using of Fourier Series with and without low pass filtering have also been performed. All methods give similar results provided that the area of the region used for interpolation (or filtering) is comparable.

\section{Path independence}

We first check the path independence of the contour integral in the cases I and II of isolated inclusions. For that purpose, we use two families of contours, square (A) and cross (B) and rectangular (C) shaped respectively as shown on Fig. 1. The size of these contours as well as their center can be varied. Figure 2 gives a summary of the results. For the three kinds of contours, we show the real and imaginary parts of the residues corresponding to Eq. (17). Note that the numerical results have been normalized according to the theoretical expectations (7) so that the expected numerical values are $\varphi_{1}=i, \psi_{1}=0$ in case I (Fig. 2 left) and $\varphi_{1}=0, \psi_{1}=1$ in case II (Fig. 2 2 right).

These numerical results can be considered as rather satisfactory in terms of orientation and orthogonality between modes $\varphi_{1}$ and $\psi_{1}$ : the measured values of quantities whose expected value is zero remain typically below $10^{-2}$. When compared to their theoretical values, $\varphi_{1}^{\text {shear }}$ and $\psi_{1}^{\text {contraction }}$ exhibit relative differences of around 5-10\% . Small fluctuations (below 5\%) can be found when changing shape and size of the contours. We already commented on the fact that the finite element simulation are performed with a single element for the inclusion, a procedure which is obviously not reliable in terms of accuracy, but which allows us to have a large ratio between element and system size.

Another test of the dependence of the numerical procedure is on the sole topology, i.e. location of the inclusion inside or outside the contour, we show in addition the dependence of the measured values of $\varphi_{1}$ and $\psi_{1}$ on the location of the contour center. Fig. 3 shows the singularity $\psi_{1}$ measured from the integration of displacement field II along a square contour centered along the $x$-axis. Results are normalized so that the expected value of $\Re \psi_{1}$ be unity when the inclusion is within the contour and zero elsewhere. The contour size is $M=20$. We obtain the expected behavior: the values of $\Re \psi_{1}$ shifts form zero to unity depending on the inclusion is within or outside the contour. Significant fluctuations (10-20\%) are however observed when the inclusion lies in the vicinity of the contour.

Finally we test the dependence of the numerical method on the material properties. In the determination of $\varphi_{1}$ and $\psi_{1}$ (17) as residues, the need to resort to a second order derivative of the displacement field is balanced by the 


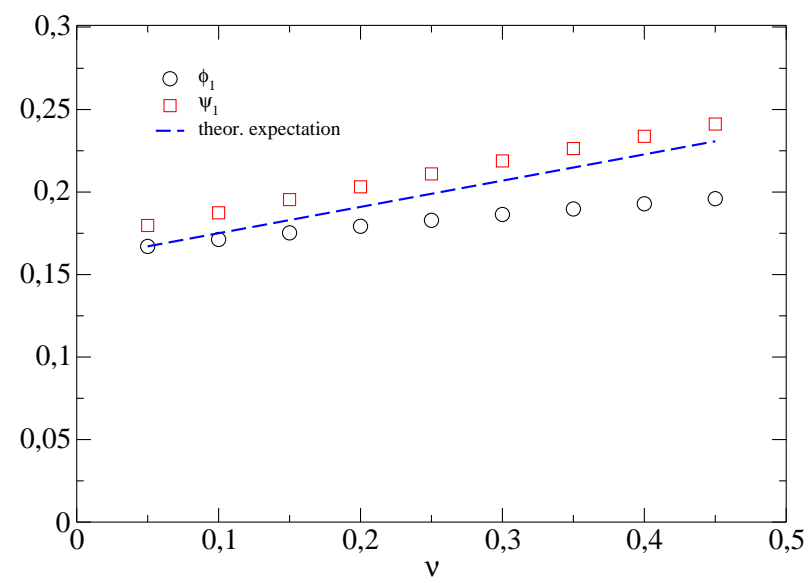

FIG. 4: Numerical estimates of $\left|\varphi_{1}\right|$ and $\psi_{1}$ obtained for a central inclusion experiencing a unit shear and a unit contraction respectively as a function of the Poisson ratio $\nu$. The numerical results obtained in plane stress conditions for a system of size $100 \times 100$ with stress free boundary conditions are compared with the theoretical expectation $(1+\nu) / 2 \pi$.

fact that the computation can be performed without any knowledge of the elastic properties of the material. The independence of the numerical procedure with the Young modulus is trivially obtained due to the linearity of the FEM computation. In Fig. 4 we show the dependence of the numerical results on the Poisson ratio. FEM computations have been performed on systems of size $100 \times 100$ with stress free boundary conditions and a central inclusion experiencing a unit shear and a unit contraction respectively. Poisson ratios have been varied from 0.05 to 0.45 by step of 0.05 . The results shown in the figure compare the numerical estimates obtained for a square contour of size 40 centered on the inclusion with the theoretical expectation $\psi_{1}=\varphi_{1}=(1+\nu) / 2 \pi$. The numerical results show that the volumetric strain is weakly dependent on Poisson's ratio, but the elementary shear is more poorly estimated for high Poisson's ratio.

\section{DISCUSSION/CONCLUSION}

The proposed approach is based on a an exact result and hence theoretically establish a parallel with other types of elastic singularities (in particular for stress intensity factors which characterize crack loadings) where similar path integral are well known. When tested on direct numerical simulations, we could recover the main topological properties expected in this context: path independence and detection of the absence/presence of a singularity within the contour. However, the quantitative results proved more disappointing: the method is rather unprecise on the determination of the prefactor of the singularity and is more generally rather sensitive to noise. The main cause is presumably due to the inconsistent regularity of the displacement field solution (simple continuity) with the need to resort to estimates of first and second order differentials. A piecewise high order polynomial interpolation is operational for integrals over finite segments, however, from one segment to the next, first and second order differentials will display a discontinuous character, which obviously affect the method and result. Moreover, being a path integral, the method does not take advantage of the knowledge of the displacement field at all points of a domain. To make the method more robust with respect to noise, different approaches can be followed. One natural way is to average the result over different contours, thus transforming the contour integral into a domain integral. An arbitrary weight average can also be considered, and hence, one could optimize the weight in order to achieve the least noise sensitivity. Such a method was explored successfully for cracks in ref. [17. Note finally that extensions to three dimensions obviously require a different technique than Kolossov and Muskhelishvili potentials, and contour integrals, however still a linear extraction operator acting on the displacement field can be computed to provide similarly the equivalent plastic strain. 


\section{Acknowledgments}

V. Petäjä acknowledges the financial support of ANR program PlastiGlass NT05-4_41640 and of the academy of Finland.

[1] V. V. Bulatov and A. S. Argon, Modell. Simul. Mater. Sci. Eng. 2, 167 (1994).

[2] V. V. Bulatov and A. S. Argon, Modell. Simul. Mater. Sci. Eng. 2, 185 (1994).

[3] V. V. Bulatov and A. S. Argon, Modell. Simul. Mater. Sci. Eng. 2, 203 (1994).

[4] M. L. Falk and J. S. Langer, Phys. Rev. E 57, 7192 (1998).

5] J.-C. Baret, D. Vandembroucq, and S. Roux, Phys. Rev. Lett. 89, 195506 (2002).

[6] G. Picard, A. Ajdari, F. Lequeux, and L. Bocquet, Phys. Rev. E 71, 010501(R) (2005).

[7] A. Lemaître and C. Caroli, arxiv:cond-mat/0609689v1 (2006).

[8] E. A. Jagla, Phys. Rev. E 76, 046119 (2007).

[9] M. Zaiser and P. Moretti, J. Stat. Mech. P08004, 79 (2005)

[10] A. Tanguy, F. Leonforte, and J.-L. Barrat, Eur. Phys. J. E 20, 355 (2006).

[11] C. E. Maloney and A. Lemaître, Phys. Rev. E 74, 016118 (2006).

[12] J. Rice, J. Appl. Mech. 35, 379 (1968).

[13] J. D. Eshelby, Proc. Roy. Soc. A 241, 376 (1957).

[14] N. Muskhelishvili, Some basic problems of the mathematical theory of elasticity (Groningen, 1953).

[15] J. Réthoré, A. Gravouil, F. Morestin, and A. Combescure, Int. J. Frac. 132, 65 (2005).

[16] S. Roux and F. Hild, Int. J. Frac. 140, 141 (2006).

[17] J. Réthoré, S. Roux, and F. Hild, Eng. Frac. Mech. in press (2008).

[18] M. Jawson and R. Bhargava, Proc. Cam. Phil. Soc. 57, 669 (1961).

[19] J. Mathiesen, I. Procaccia, and I. Regev, arxiv:0704.1206v1 (2007).

[20] C. Goldenberg and I. Goldhirsch, Eur. Phys. J. E 9, 245 (2002). 